\title{
ASPECTOS ÉTICOS NO EXERCÍCIO DA ENFERMAGEM: REVISÃO INTEGRATIVA DA LITERATURA
}

Karelline Izaltemberg Vasconcelos Rosenstock ${ }^{1}$, Maria Julia Guimarães Oliveira Soares ${ }^{2}$, Sérgio Ribeiro dos Santos ${ }^{3}$, Anayde Selma Marcelino Ferreira ${ }^{4}$

RESUMO: Este estudo objetivou identificar, a partir da revisão integrativa da literatura, a produção científica on-line nacional e internacional sobre o tema Ética na Enfermagem. Foram analisados 53 artigos científicos publicados no período de 2001 a 2010. A busca dos artigos foi empreendida nos bancos de dados da Biblioteca Virtual em Saúde e no portal de periódicos da Coordenação de Aperfeiçoamento de Pessoal de Nível Superior. A pesquisa revelou que 69,8\% dos artigos publicados é produção nacional sobre o tema, indicando boas perspectivas para as reflexões éticas no campo assistencial da saúde e na formação acadêmica dos profissionais de Enfermagem. Entretanto, a produção ainda é incipiente em relação a esta temática, sendo necessário o desenvolvimento de estudos e pesquisas para maior aprofundamento neste campo. A incorporação de aspectos éticos e legais na vivência profissional do enfermeiro é imprescindível para uma assistência de Enfermagem integral e de qualidade.

PALAVRAS-CHAVE: Ética em enfermagem; Legislação de enfermagem; Prática profissional; Enfermagem.

\section{ETHICAL ASPECTS IN THE EXERCISING OF NURSING: AN INTEGRATIVE LITERATURE REVIEW}

ABSTRACT: Through an integrative review of the literature this study aimed to identify Brazilian and international on-line scientific production on the subject of Ethics in Nursing. Fifty-three scientific articles published in the period $2001-2010$ were analyzed. The article search was undertaken in the databank of the Virtual Health Library and in the website of the Coordination for Perfection of Higher Education Personnel. The survey showed that $69.8 \%$ of the articles published on the theme are Brazilian, indicating a good outlook for ethical reflection in the area of health care and in the academic preparation of Nursing professionals. Production, however, remains incipient in relation to this thematic, the development of studies and research for greater deepening of this field being necessary. The incorporation of ethical and legal aspects into the professional experience of the nurse is crucial for quality integral nursing care.

KEYWORDS: Ethics in nursing; Nursing legislation; Professional practice; Nursing.

\section{ASPECTOS ÉTICOS EN EL EJERCICIO DE LA ENFERMERÍA: REVISIÓN INTEGRATIVA DE LA LITERATURA}

RESUMEN: Este estudio tuvo el propósito de identificar, con base en la revisión integrativa de la literatura, la producción científica on-line nacional e internacional sobre el tema Ética en la Enfermería. Fueron analizados 53 artículos científicos publicados en el período de 2001 a 2010. La búsqueda de los artículos ocurrió en los bancos de datos de la Biblioteca Virtual en Salud y en el portal de periódicos de la Coordinación de Perfeccionamiento de Personal de Nivel Superior. La investigación ha revelado que $69,8 \%$ de los artículos publicados son producción nacional acerca del tema, indicando buenas perspectivas para las reflexiones éticas en el campo asistencial de la salud y en la formación académica de los profesionales de Enfermería. Sin embargo, la producción todavía es incipiente en relación a esta temática, siendo necesario el desarrollo de estudios e investigaciones para mayor profundización en este campo. La incorporación de aspectos éticos y legales en la experiencia profesional del enfermero es imprescindible para una asistencia de Enfermería integral y de cualidad. PALABRAS-CLAVE: Ética en enfermería; Legislación de enfermería; Práctica profesional; Enfermería.

\footnotetext{
${ }^{1}$ Enfermeira. Mestre em Enfermagem. Professora do Curso de Graduação em Enfermagem da Universidade Federal da Paraíba-UFPB. Membro do Grupo de Estudos e Pesquisa em Administração e Informática em Saúde-GEPAIE.

${ }^{2}$ Enfermeira. Doutora em Enfermagem. Professora do Curso de Graduação e do Programa de Pós-Graduação em Enfermagem da UFPB. Líder do Grupo de Estudos e Pesquisa no Tratamento de Feridas.

${ }^{3}$ Enfermeiro. Doutor em Sociologia. Professor do Programa de Pós-Graduação em Enfermagem da UFPB. Líder do GEPAIE.

${ }^{4}$ Enfermeira. Membro do GEPAIE.
}

Autor correspondente:

Recebido: $12 / 03 / 2011$

Karelline Izaltemberg Vasconcelos Rosenstock

Aprovado: $31 / 08 / 2011$

Universidade Federal da Paraíba

Rua José Rufino, 393 - 58046-080 - João Pessoa-PB-Brasil

E-mail: mrosenstock@ig.com.br 


\section{INTRODUÇÃO}

Atualmente, a preocupação com a ética vem emergindo de maneira marcante em nossa sociedade, mais precisamente no âmbito da saúde, devido às novas descobertas e experimentos científicos. A Enfermagem vem aprofundando suas reflexões e questionamentos sobre a sua prática, a fim de enfrentar esses desafios, bem como as questões éticas que cotidianamente surgem em seu campo de atuação.

O trabalho de Enfermagem tem se diversificado, indo desde o cuidado, seja do indivíduo, família e grupo da comunidade, passando pelas ações educativas, pesquisas, administrativas, até a participação no planejamento em saúde. O enfermeiro tem avançado no controle das suas atividades, previstas tanto no Regulamento do Exercício Profissional (Lei n. 7498/86) $)^{(1)}$ como no Código de Ética dos Profissionais de Enfermagem (Resolução COFEN 311/07) ${ }^{(2)}$. Surgem, junto com este avanço, os problemas éticos relativos ao desempenho da profissão de Enfermagem, sendo necessário que esse profissional tome conhecimento de seus direitos e deveres éticos e legais, ampliando, assim, a segurança em suas ações e a possibilidade de estar exercendo as suas atividades dentro daquilo que lhe cabe, evitando possíveis complicações legais posteriores ${ }^{(2)}$.

A ética profissional é uma parte da ciência moral e tem como função detectar os fatores que, numa determinada sociedade, são capazes de alienar a atividade profissional; portanto, é tarefa da ética profissional realizar uma reflexão crítica, e questionadora, que tenha por finalidade salvar e dar segurança à sociedade no que diz respeito à atividade profissional ${ }^{(3)}$.

A ética, no contexto da Enfermagem, abrange comportamentos e ações que envolvem conhecimentos, valores, habilidades e atitudes no sentido de favorecer as potencialidades do ser humano com a finalidade de manter ou melhorar a condição humana no processo de viver e morrer. Assim, os cuidados de Enfermagem devem estar relacionados a ações livres de danos decorrentes de imperícia, negligência ou imprudência ${ }^{(4)}$.

Diante do exposto, percebemos que a ética é difícil de ser vivenciada, disseminada e divulgada na prática, uma vez que muitos valores estão envolvidos nesse contexto, tais como valores culturais, sociais e religiosos que vêm de encontro ao caráter do indivíduo ${ }^{(5)}$. Contudo, observa-se que a literatura internacional e nacional que trata desse tema é merecedora de estudo, sendo necessário investigar trabalhos inerentes à temática para uma melhor compreensão do fenômeno, tendo em vista que a grande maioria dos profissionais desconhece o código de ética da profissão, dificultando o exercício pleno de uma prática profissional pautada em aspectos éticos e legais.

Assim, os objetivos deste artigo são: identificar a produção científica online nacional e internacional no campo da Enfermagem, sobre o tema Ética na Enfermagem, no período compreendido entre 2001 a 2010; verificar os delineamento dos estudos referenciados nas publicações; averiguar a metodologia utilizada e investigar os enfoques sobre a temática contemplados nas publicações selecionadas para pesquisa.

\section{METODOLOGIA}

Trata-se de uma revisão integrativa, a qual consiste na construção de uma análise ampla da literatura, contribuindo para discussões sobre resultados de pesquisas, assim como, reflexões sobre a realização de futuros estudos, obtendo um profundo entendimento de um determinado fenômeno com base em estudos anteriores $^{(6)}$. As etapas desenvolvidas foram as seguintes: identificação do tema e seleção da questão de pesquisa para a elaboração da revisão integrativa; estabelecimento de critérios para inclusão e exclusão de estudos e busca na literatura; definição das informações a serem extraídas dos estudos selecionados e categorização dos estudos; avaliação dos estudos incluídos na revisão integrativa; interpretação dos resultados; e, por fim, a apresentação da revisão/síntese do conhecimento ${ }^{(7)}$.

Para delineamento do estudo procuramos respostas para as seguintes questões norteadoras: Como se configura a produção científica sobre o tema Ética na Enfermagem? Qual o enfoque dado à temática pelas publicações atuais?

A pesquisa do material empírico foi desenvolvida no mês de maio de 2010. As buscas foram realizadas online na Biblioteca Virtual em Saúde (BVS), nas seguintes bases de dados indexadas: Scientific Eletronic Library Online (SciELO), Literatura Latino-Americana e do Caribe em Ciências da Saúde (LILACS), BIREME, Literatura Internacional em Ciências da Saúde (MEDLINE) e Acervo da Biblioteca da Organização Pan-americana da Saúde (PAHO); no portal de periódicos da Coordenação de Aperfeiçoamento de Pessoal de Nível Superior (CAPES), onde foram investigadas as bases de dados SpringerLink, Mary Ann Liebert, Oxford Journals, Wiley InterScience e Highwire Press. 
A busca bibliográfica foi realizada a partir da integração dos seguintes descritores encontrados no DeCS, nos idiomas português, inglês e espanhol: Ética em Enfermagem $O R$ Legislação de Enfermagem $O R$ Prática Profissional $O R$ Ética profissional $O R$ Prática de Enfermagem $O R$ Ethics Nursing $O R$ Legislation Nursing $O R$ Ética en Enfermería $O R$ Legislación de Enfermería.

Os critérios para a seleção da amostra foram: que o estudo abordasse, no título ou no resumo, a temática investigada; que a publicação estivesse no intervalo entre 2001 a maio de 2010; que o artigo apresentasse o texto na íntegra e nos idiomas português, inglês e espanhol.

O resultado da busca na BVS foi de 239 estudos, 159 abordavam o tema Ética na Enfermagem, mas apenas 37 apresentavam o texto na íntegra, dos quais 18 indexados na SciELO, 7 na MEDLINE, 5 na LILACS, 4 na BIREME e 3 na PAHO. No portal de periódicos da CAPES foram encontrados 211 estudos, mas apenas 31 estavam disponíveis, sendo que 15 publicações não se referiam à temática, restando apenas 16 trabalhos, dos quais 4 indexados na SpringerLink, 4 na Highwire Press, 3 na Oxford Journals, 3 na Wiley InterScience e 2 na Mary Ann Liebert. Assim, no total, 53 publicações fizeram parte da amostra.

Para organização das informações contidas nas publicações encontradas foi utilizado um instrumento para a coleta de dados, contendo título, ano e natureza da publicação; objetivos, metodologia e as considerações finais dos trabalhos. Para análise dos dados coletados, este estudo utilizou a técnica de análise de conteúdo, definida como um conjunto de técnicas de análise de comunicação "visando a obter [...] indicadores (quantitativos ou não) que permitam a inferência de conhecimentos relativos às condições de produção/recepção destas mensagens" ${ }^{\prime(8: 15)}$.

A técnica de análise de conteúdo é composta por três etapas: 1) a pré-análise, onde é realizada a organização e leitura flutuante do material; 2) a exploração do material, com codificação das unidades de registro; e 3) o tratamento dos resultados, interpretação e categorização dos conteúdos ${ }^{(8)}$. Assim, para alcançar os objetivos propostos, os conteúdos temáticos encontrados nos trabalhos foram classificados nas seguintes categorias: Ética e o Cuidar em Enfermagem, Dilemas Éticos, Código de Ética dos Profissionais de Enfermagem, Ética no Exercício Profissional da Enfermagem, Ética na Pesquisa em Enfermagem e Ensino da Ética.

\section{RESULTADOS}

O material de análise do estudo proposto foi constituído por 53 artigos, publicados no período de 2001 a maio de 2010, e que tomavam a questão da Ética na Enfermagem como tema central. Os artigos encontrados evidenciam que a maioria das publicações é nacional (38 artigos) representando $69,8 \%$ da amostra. Diante disso, percebe-se a grande importância do tema no Brasil.

Quanto ao ano de publicação, obtivemos os seguintes resultados: no biênio 2001-2002 foram três artigos publicados (5,7\%), em 2003-2004 onze artigos (20,8\%), em 2005-2006 dezessete artigos (32,1\%), em 2007-2008 doze (22,6\%) e em 2009-2010 constataram-se dez artigos $(18,9 \%)$. É importante destacar que, no biênio de 2005-2006 evidenciou-se uma expressiva produção. Com relação ao delineamento dos artigos investigados houve uma predominância de artigos de revisão $(35,8 \%)$ e artigos originais (28,3\%), ambos os grupo de produção nacional. Quanto ao tipo de estudo, verificou-se que prevaleceram revisões de literatura $(45,3 \%)$ e pesquisas qualitativas $(18,9 \%)$, conforme mostra a tabela 1 .

Tabela 1 - Distribuição dos artigos segundootipo de estudo, 2001-2010

\begin{tabular}{lcc}
\hline \multicolumn{1}{c}{ Tipo de estudo } & Frequência & $\mathbf{\%}$ \\
\hline Pesquisa qualitativa & 10 & 18,9 \\
Pesquisa quantitativa & 3 & 5,7 \\
Revisão da literatura & 24 & 45,3 \\
Estudo de caso & 1 & 1,9 \\
Pesquisa quantiqualitativa & 5 & 9,4 \\
Meta-análise & 1 & 1,9 \\
Fenomenologia social & 1 & 1,9 \\
Relato de experiência & 3 & 5,7 \\
Pesquisa documental & 4 & 7,5 \\
Revisão sistemática & 1 & 1,9 \\
\hline \multicolumn{1}{c}{ Total } & $\mathbf{5 3}$ & $\mathbf{1 0 0}$ \\
\hline
\end{tabular}

Quanto às categorias propostas para o estudo, verifica-se que a categoria Dilemas Éticos representa 8 artigos $(15,1 \%)$ que retratam situações onde há uma tomada de decisão envolvendo princípios éticos; a categoria denominada Ética e o Cuidar em Enfermagem compreende $11(20,8 \%)$ publicações que abordam a relação ética-cuidado na assistência de Enfermagem.

Com relação ao Código de Ética dos Profissionais de Enfermagem são destacados nessa categoria 4 trabalhos $(7,5 \%)$ que abordam a importância da regulamentação e controle do exercício profissional da Enfermagem; na categoria Ética no Exercício Profissional da Enfermagem, que compreende a maior parte 
dos artigos, 16 trabalhos (30,2\%) abordam os aspectos éticos relacionados à prática da profissão. A categoria nomeada de Ética na Pesquisa em Enfermagem inclui 7 artigos (13,2\%) que versam sobre as atividades dos Comitês de Ética em Pesquisa e a necessidade de controle das pesquisas em Enfermagem; e a categoria Ensino da Ética apresenta também 7 publicações $(13,2 \%)$ que versam sobre a importância do ensino da ética na formação do profissional de Enfermagem.

\section{DISCUSSÃO}

No que concerne aos enfoques da categoria Dilemas Éticos, os artigos deram grande ênfase às situações que envolvem decisão relacionada com princípio ético ou prática moral. Um dilema ético é uma situação onde o indivíduo pode ver dois lados de um problema, sem que exista opção moral claramente certa ou errada que justifique decisão óbvia ${ }^{(9)}$.

Os artigos destacam os dilemas e conflitos éticos associados à assistência de Enfermagem, ao diagnóstico de Enfermagem, ao código de ética e a formação ética do enfermeiro ${ }^{(10-14)}$. Alguns artigos assinalam que a assistência à saúde é passível de questões e dilemas éticos, que, se não resolvidos adequadamente, podem comprometer a atuação profissional, a qualidade do atendimento e a autonomia do paciente ${ }^{(15-16)}$.

Estudos apontam que muitos enfermeiros vêm experimentando novas exigências éticas impostas por uma sociedade multicultural que, quando confrontados com dilemas éticos tendem a usar as convenções como orientação predominante dos seus critérios de decisão e não as necessidades dos pacientes e seu bemestar $^{(11-12)}$. Esta prática de seguir as convenções em vez de perseguir o melhor para o paciente constitui um obstáculo importante para o enfermeiro assumir uma postura ética adequada nas suas ações. Nessa perspectiva, diversos profissionais, diante dos dilemas éticos decorrentes, demonstram que estão cada vez mais à procura de respostas que lhes assegurem uma dimensão humana nas relações profissionais, principalmente aquelas associadas à autonomia, justiça e necessidade de respeito à dignidade da pessoa humana ${ }^{(16)}$.

Na categoria Ética e o Cuidar em Enfermagem, os artigos abordam a relação ética - cuidado na assistência de Enfermagem; grande parte dos autores afirma que os enfermeiros têm interesse em garantir a qualidade do processo de cuidar com segurança e benefício para o paciente ${ }^{(17-19)}$. Os princípios éticos são uma referência importante na assistência de Enfermagem: o princípio da beneficência, fazer o bem; da não-maleficência, primeiramente não fazer o mal; da justiça, distribuição justa, equitativa e apropriada; e o princípio da autonomia, autodeterminação ou autogoverno, poder de decidir sobre si mesmo ${ }^{(19-21)}$.

Inspirado pela dignidade da pessoa e seu direito inviolável de ser, o enfermeiro precisa agir na defesa da autonomia e no respeito pelas opções da pessoa quanto ao seu cuidado. Desta forma, a base do conhecimento em Enfermagem ética é o sentido moral, à medida que a Enfermagem se esforça para delinear a sua própria teoria ética, e o cuidado é citado frequentemente como a base moral ${ }^{(22-23)}$. Os cuidados de Enfermagem devem ser desenvolvidos com base no respeito pela vida, dignidade e direitos humanos, garantindo a liberdade da pessoa humana e evocando a qualidade da assistência à saúde. A dimensão ética assume grande relevância no processo de tomada de decisão; sua utilização por parte dos enfermeiros nos seus comportamentos profissionais torna-se imprescindível ${ }^{(19)}$.

No enfoque da categoria Código de Ética dos Profissionais de Enfermagem, todos os artigos encontrados são internacionais e abordam a importância da regulamentação e controle do exercício profissional da Enfermagem $^{(24-26)}$. Os artigos apontam que vivemos, atualmente, um novo contexto profissional, portanto, os códigos profissionais de Enfermagem precisam se adequar às crescentes exigências éticas colocadas para a profissão, reavaliando os seus objetivos morais ${ }^{(24-25)}$.

Os artigos internacionais propõem um novo código de ética para a Enfermagem, com o papel de cumprir várias funções, incluindo o apoio à sua identidade profissional (função externa) e as orientações para a prática de Enfermagem (função interna) ${ }^{(25-26)}$. Em uma publicação, os autores acreditam que a prática ampliada da Enfermagem apresenta preocupações éticas que não são abordados pelos códigos vigentes ${ }^{(25)}$. O Código de Ética dos Profissionais de Enfermagem do Brasil, em sua última edição, de 2007, é uma boa manifestação dos princípios da formação profissional; ser capaz de aplicar esses princípios pode influenciar a satisfação no trabalho da Enfermagem, promovendo uma melhora na qualidade do cuidado prestado.

Na categoria Ética no Exercício Profissional da Enfermagem, os artigos abordam os aspectos éticos relacionados à prática da profissão. Os artigos enfatizam que a atuação ética da Enfermagem perpassa a humanização da assistência, a melhoria contínua do processo de cuidar, a credibilidade profissional, a satisfação da clientela, a parceria no processo educativo e o respeito ao sigilo ético ${ }^{(27-29)}$. 
Alguns artigos abordam os conflitos morais vivenciados pelos trabalhadores de Enfermagem, apontando que estes problemas podem lhes provocar sofrimento moral ${ }^{(30-32)}$. Pode-se inferir que para o exercício saudável da Enfermagem algumas atitudes são fundamentais: o diálogo aberto entre a chefia e os enfermeiros, no sentido de buscar medidas para evitar frustrações, prevendo mudanças; maior envolvimento dos enfermeiros no desenvolvimento de políticas organizacionais que valorizem a dimensão humana e social; encorajamento para participar dos comitês de ética; presença de educação permanente que fortaleça as escolhas éticas; e esclarecimento contínuo de valores, tendo como parâmetros o Código de Ética dos Profissionais da Enfermagem.

A categoria Ética na Pesquisa em Enfermagem inclui artigos que versam sobre as atividades dos Comitês de Ética em Pesquisa e a necessidade de controle das pesquisas em Enfermagem. Os artigos relatam que é crescente a preocupação dos pesquisadores quanto à necessidade de avaliar a qualidade e o alcance das pesquisas em Enfermagem. A apresentação de um projeto de pesquisa à apreciação de um Comitê de Ética em Pesquisa é parte essencial e inicial do processo de produção do conhecimento científico ${ }^{(33-35)}$. Nesta perspectiva, a Enfermagem tem o desafio de cuidar e pesquisar para implementação do conteúdo teórico-científico baseado em resultados de pesquisas evidenciadas na prática da investigação.

Além disso, os artigos apontam que no campo da Enfermagem as investigações, em sua maior parte, são realizadas com seres humanos, tornando-se necessário que o pesquisador atenda ao cumprimento dos princípios éticos contidos na Declaração de Helsinki de 1964, reformulada em 2008, bem como resoluções específicas de cada país, como no caso do Brasil, onde a Resolução n. 196/96 determina as exigências éticas a serem adotadas em todas as pesquisas envolvendo seres humanos ${ }^{(36-37)}$.

A pesquisa empírica procura descobrir os conhecimentos incorporados na prática, enriquecendo e melhorando a ética. A integração da pesquisa com a prática profissional leva à melhor compreensão teórica da natureza multidimensional da realidade. Desta forma, é a interação dinâmica entre as duas disciplinas que contribui para uma melhor compreensão teórica e empírica das questões éticas e, consequentemente, para um melhor atendimento ao paciente ${ }^{(37)}$.

Na categoria Ensino da Ética estão retratados os artigos que relatam a importância do ensino da ética na formação do profissional de Enfermagem. Os autores atentam para a necessidade de redirecionar o ensino da ética em Enfermagem, de um enfoque deontológico, centrado em discussões conceituais, para um ensino transversal de valores, por meio de metodologias ativas, a fim de que haja a formação de uma consciência ética que ultrapasse as necessidades da profissão, pois satisfaz uma necessidade social e cultural da urgência de se retomar o significado dos valores morais ${ }^{(38-40)}$.

Para tanto, faz-se necessário exigir de todos os envolvidos no processo ensino-aprendizagem uma postura inovadora, que desencadeie uma nova prática. Mas, para que isso se concretize, é preciso um conhecimento fundamental do ser humano, oportunizando ao discente, futuro profissional, experiências significativas; liberdade para pensar e questionar; desenvolvimento de ações interativas, humanas e solidárias; e espaço para atuar com autonomia, a fim de desenvolver seu potencial crítico-reflexivo, tendo como núcleo da sua formação como enfermeiro a pessoa humana ${ }^{(39)}$.

\section{CONSIDERAÇÕES FINAIS}

A pesquisa revelou a necessidade de desenvolvimento de novas pesquisas sobre a Ética na Enfermagem que levem a um aumento da produção científica em nível nacional e internacional. A partir do estudo, foi possível identificar que há expressiva produção de artigos nacionais em relação à produção internacional incluídas na amostra; esse resultado pode ter sido em decorrência da estratégia de inclusão dos artigos no estudo.

Os resultados evidenciam a atenção dos pesquisadores quanto à necessidade da formulação de um perfil ético do profissional de Enfermagem que atenda às várias possibilidades e que seja, ao mesmo tempo, comprometido com o cuidado e o bem-estar das pessoas. Esta preocupação com o tema indica boas perspectivas para se inserir reflexões éticas no campo assistencial da saúde e na formação acadêmica dos profissionais de Enfermagem. Entretanto, destaca-se que a produção ainda é incipiente em relação à temática, sendo necessário que os pesquisadores desenvolvam estudos e pesquisas para que se avance no debate e no levantamento de questões éticas relacionadas à prática da Enfermagem.

As discussões apontam a relevância da ética e da moral nas representações sociais, a fim de alcançar o aperfeiçoamento e a humanização da assistência prestada ao paciente, sendo a Ética uma nova proposta na análise das questões morais dos grupos sociais. O conhecimento e a compreensão da ética permitem ao enfermeiro exercer com competência, resolutividade e segurança o seu papel profissional na sociedade. O exercício da Enfermagem 
deve estar baseado em valores morais da profissão, em seu código de ética e nos direitos do paciente hospitalizado, integrando cuidado humano qualificado, guiado pelo respeito e pela promoção do paciente como um protagonista e sujeito do cuidado. Conclui-se, portanto, que é imprescindível a incorporação de aspectos éticos e legais na vivência profissional do enfermeiro, contribuindo para uma assistência completa e de qualidade.

\section{REFERÊNCIAS}

1. Brasil. Lei 7.498, de 25 de junho de 1986. Dispõe sobre a Regulamentação do Exercício da Enfermagem e dá outras providências. Brasília: Ministério da Saúde; 1986. [acesso em 15 mai 2010]. Disponível: http://www. planalto.gov.br/ccivil_03/leis/L7498.htm

2. Conselho Federal de Enfermagem. Código de ética dos profissionais de enfermagem. Rio de Janeiro: COFEN; 2008.

3. Trautman DA. Educação, ética e tecnologia: impressões e reflexões [dissertação]. Florianópolis (SC): Universidade Federal de Santa Catarina; 2002.

4. Waldow VR. Cuidado humano: o resgate necessário. Porto Alegre: Sagra Luzzatto; 2001.

5. Freire P. Pedagogia da autonomia: saberes necessários à prática educativa. São Paulo: Paz e Terra; 2005.

6. Broome ME. Integrative literature reviews for the development of concepts. In: Rodgers BL, Knafl KA, editors. Concept development in nursing: foundations, techniques and applications. Philadelphia (USA): WB Saunders Company; 2000. p. 231-50.

7. Ganong LH. Integrative reviews of nursing research. Res Nurs Health. 1987;10(1):1-11.

8. Bardin L. Análise de conteúdo. $20^{\mathrm{a}}$ ed. Lisboa: Edições 70; 2009 .

9. Robertson CJ, Crittenden W, Brady MK, Hoffman JJ. Situational ethics across borders: a multicultural examination. J Business Ethics. 2002;38(4):327-38.

10. Beverly K, Burkhart L. Ethical dilemma and moral distress: proposed new NANDA diagnoses. Int J Nurs Terminol Classif . 2005;16(1):3-13.

11. Fakih FT, Freitas GF, Secoli SR. Medicação: aspectos ético-legais no âmbito da enfermagem. Rev Bras Enferm. 2009;62(1):132-5.
12. Lima EDRP, Magalhaes MBB, Nakamae DD. Aspectos ético-legais da retirada e transplante de tecidos, órgãos e partes do corpo humano. Rev Latino-Am Enfermagem. 2007;5(4):5-12.

13. Monteiro MAA, Barbosa RCM, Barroso MGT, Vieira NFC, Pinheiro AKB. Dilemas éticos vivenciados por enfermeiros apresentados em publicações de enfermagem. Rev Latino-Am Enfermagem. 2008;16(6):121-7.

14. Franca ISX, Baptista RS, Brito VRS. Dilemas éticos na hemotransfusão em testemunhas de Jeová: uma análise jurídico-bioética. Acta Paul Enferm. 2008;21(3):498-503.

15. Biton V, Taba N. The relationship between the application of the nursing ethical code and nurses' work satisfaction. Int J Nurs Pract. 2003;(9):140-57.

16. Leite AIT, Claudino HG, Santos SR dos. A importância de ser ético: da teoria à prática de enfermagem. Cogitare Enferm. 2009;14(1):172-7.

17. Noureddine S. Development of the ethical dimension in nursing theory. Int J Nurs Pract. 2001;7(1):2-7.

18. Zoboli ELCP. A redescoberta da ética do cuidado: o foco e a ênfase nas relações. Rev Esc Enferm USP. 2004;38(1):21-7.

19. Mendes G. A dimensão ética do agir e as questões da qualidade colocadas face aos cuidados de enfermagem. Texto \& Contexto Enferm. 2009;18(1):165-9 .

20. Bellato R, Pereira WR. Direitos e vulnerabilidade: noções a serem exploradas para uma nova abordagem ética na enfermagem. Texto \& Contexto Enferm. 2005;14(1):17-24.

21. Koerich MS, Machado RR, Costa E. Ética e bioética: para dar início à reflexão. Texto \& Contexto Enferm. 2005;14(1):106-10.

22. Tarlier DS. Beyond caring: the moral and ethical bases of responsive nurse-patient relationships. Philos Nurs. 2004;5(3):230-41.

23. Volker DL. Is there a unique nursing ethic? Nurs Sci Q. 2003;16(3):207-11.

24. Meulenbergs T, Verpeet E, Schotsmans P, Gastmans C. Professional codes in a changing nursing context: literature review. J Adv Nurs. 2004;46(3):331-6.

25. Peterson M, Potter RL. A proposal for a code of ethics for nurse practitioners. J Am Pract. 2004;16(3):116-24. 
26. Verpeet E, Dierckx CB, Van der Arend A, Gastmans CAE. Nurses' views on ethical codes: a focus group study. J Adv Nurs. 2005;51(2):188-95.

27. Swanson KM, Wojnar DM. Optimal healing environments in nursing. J Altern Complement Med. 2004;10 Suppl 1:43-8.

28. Zoboli ELCP. Enfermeiros e usuários do programa saúde da família: contribuições da bioética para reorientar esta relação profissional. Acta Paul Enferm. 2007;20(3):16-20

29. Maidana S. La dimensión ética del trabajo. Texto Contexto Enferm. 2005;14(1):58-64.

30. Wendhausen ÁLP, Rivera S. O cuidado de si como princípio ético do trabalho em Enfermagem. Texto Contexto Enferm. 2005;14(1):111-09.

31. Liaschenko J, Peter E. Nursing ethics and conceptualizations of nursing: profession, practice and work. J Adv Nurs. 2004;46(5):488-95.

32. Lunardi VL, Barlem ELD, Bulhosa MS, Santos SC, Lunardi Filho WD, Silveira RS, et al. Sofrimento moral e a dimensão ética no trabalho da enfermagem. Rev Bras Enferm. 2009;62(1):599-603.

33. Ducati C, Boemer MR. Comissões de ética de enfermagem em instituições de saúde de Ribeirão Preto. Rev Latino-Am Enfermagem. [Internet] 2001;9(3) [acesso em 15 mai 2010]. Disponível: http://www.scielo.br/pdf/rlae/v9n3/11495.pdf

34. Massarollo MCKB, Kurcgant P, Fernandes MFP. Comitê de ética em pesquisa da escola de enfermagem da USP: experiência dos primeiros seis anos. Rev Esc Enferm USP. 2009;43(n. esp 2):1303-7.

35. Dierckx CB, Grypdonck M, N Cannaerts, Steeman E. Empirical ethics in action: lessons from two empirical studies in nursing ethics. Med Health Care Philos. 2004;7:31-9.

36. Ferreira HM, Ramos LH. Diretrizes curriculares para o ensino da ética na graduação em enfermagem. Acta Paul Enferm. 2006;19(3):328-31.

37. Furukawa PO, Cunha ICKO. Comitês de ética em pesquisa: desafios na submissão e avaliação de projetos científicos. Rev Bras Enferm. 2010;63(1):145-7.

38. Santiago MMA, Palacios M. Temas éticos e bioéticos que inquietaram a enfermagem: publicações da REBEn de 1970-2000. Rev Bras Enferm. 2009;59(3):349-53.
39. Cruz EA, Alves MDS, Fraga MNO, Damasceno MMC. Abordagem ética em pesquisas publicadas por um programa de pós-graduação em enfermagem. Texto Contexto Enferm. 2005;14(1):25-32.

40. Fernandes JD, Rosa DOS, Vieira TT, Sadigursky D. Dimensão ética do fazer cotidiano no processo de formação do enfermeiro. Rev Esc Enferm USP. 2008;42(2):396-403. 